\title{
The Prognostic Impact of Tumor Differentiation on Recurrence and Survival after Resection of Hepatocellular Carcinoma Is Dependent on Tumor Size
}

\author{
Hiroji Shinkawa ${ }^{a}$ Shogo Tanaka ${ }^{a}$ Daijiro Kabata $^{b}$ Shigekazu Takemura ${ }^{a}$ \\ Ryosuke Amano $^{a}$ Kenjiro Kimura ${ }^{a}$ Masahiko Kinoshita ${ }^{a}$ Shoji Kubo ${ }^{a}$ \\ aDepartment of Hepato-Biliary-Pancreatic Surgery, Osaka City University Graduate School of Medicine,

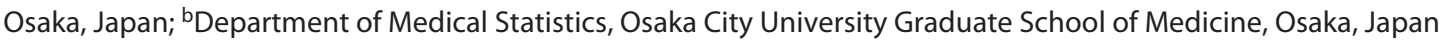

\section{Keywords}

Tumor differentiation $\cdot$ Hepatocellular carcinoma $\cdot$ Tumor size $\cdot$ Prognosis

\begin{abstract}
Introduction: The present study aimed to evaluate the effect of poor differentiation and tumor size on survival outcome after hepatic resection of hepatocellular carcinoma (HCC). Methods: A total of 1,107 patients who underwent initial and curative hepatic resection for HCC without macroscopic vascular invasion participated in the study. Using the multivariable Cox proportional hazards regression model, we evaluated changes in hazard ratios (HRs) for the association between tumor differentiation and survival based on tumor size. Results: In patients with poorly (Por) differentiated HCCs, the adjusted HRs of reduced overall survival (OS), recurrence-free survival (RFS), early RFS, and early extrahepatic RFS were 1.31 (95\% confidence interval [Cl]; 1.07-1.59), 1.07 (95\% Cl 0.89-1.28), 1.31 (95\% Cl 1.06$1.62)$, and 1.81 (95\% Cl 1.03-3.17), respectively. Moreover,
\end{abstract}

based on an analysis of the effect modification of tumor differentiation according to tumor size, Por HCC was found to be associated with a reduced OS ( $p=0.033)$. The HRs of Por HCCs sharply increased in patients with tumors measuring up to $5 \mathrm{~cm}$. The adjusted HRs of reduced OS in patients with Por HCCs measuring $<2, \geq 2$ and $<5$, and $\geq 5 \mathrm{~cm}$ were 1.22 (95\% Cl 0.69-2.14), 1.33 (95\% Cl 1.02-1.73), and 1.58 (95\% Cl 1.04-2.42), respectively. The corresponding adjusted HRs of reduced early RFS were 0.85 ( $95 \% \mathrm{Cl} 0.46-1.57), 1.34$ (95\% $\mathrm{Cl} 1.01-1.8)$, and 1.57 (95\% Cl 1.03-2.39), respectively. The adjusted HRs of reduced early extrahepatic RFS were 1.89 $(95 \% \mathrm{Cl} 0.83-4.3)$ in patients with tumors measuring $\geq 2$ and $<5 \mathrm{~cm}$ and 2.33 (95\% Cl 0.98-5.54) in those with tumors measuring $\geq 5 \mathrm{~cm}$. Conclusions: Por HCC measuring $\geq 2 \mathrm{~cm}$ was associated with early recurrence. Hence, it had negative effects on OS. After surgery, patients with Por HCC measuring $\geq 5 \mathrm{~cm}$ should be cautiously monitored for early extrahepatic recurrence. These findings will help physicians devise treatment strategies for patients with HCC.

(c) 2021 The Author(s)

Published by S. Karger AG, Basel karger@karger.com www.karger.com/lic

Karger $\stackrel{\text { ' }}{5}$

GOPEN ACCESS
(C) 2021 The Author(s)

Published by S. Karger AG, Basel

This is an Open Access article licensed under the Creative Commons Attribution-NonCommercial-4.0 International License (CC BY-NC) (http://www.karger.com/Services/OpenAccessLicense), applicable to the online version of the article only. Usage and distribution for commercial purposes requires written permission.
Correspondence to:

Hiroji Shinkawa, m1297198@ msic.med.osaka-cu.ac.jp 


\section{Introduction}

Hepatocellular carcinoma (HCC) is one of the leading causes of cancer-related death worldwide [1]. Despite improvements in the clinical management of patients with HCC, the prognosis remains unsatisfactory. Several clinicopathological factors related to background liver disease and tumor progression have been reported to predict poor outcome [2-4]. Oncological characteristics with regard to tumor malignancy and aggressiveness obtained from pathological findings are considered prognostic factors for HCC [5-7]. Tumor differentiation is determined by cytological atypia and growth pattern. Poorly (Por) differentiated HCC with marked cellular pleomorphism is considered malignant, exhibiting invasiveness and a tendency to metastasize [8-10]. Por HCC is thought to have a potentially negative effect on long-term survival; however, many studies have shown no significant effect of tumor differentiation on prognosis [11-13]. The European Association for the Study of the Liver guidelines indicated that the prognostic value of tumor differentiation would be equivocal [5].

Previous studies have demonstrated that the frequency of poor differentiation increased as tumor size increased $[12,14]$. The frequency of microscopic vascular invasion by Por HCC was also reported to increase with tumor size [14]. Meanwhile, prognostic effects of malignant characteristics of HCC, such as microvascular invasion and increased serum $\alpha$-fetoprotein level (a tumor biomarker), were reported to be influenced by tumor size, but the predictive value of both characteristics for survival was not identified in HCCs that were small $(<2 \mathrm{~cm}$ in size) [15]. However, no investigations have focused on whether the prognostic effect of tumor differentiation on survival outcomes is modified by tumor size. To clarify the prognostic value of tumor differentiation in patients with HCC, we investigated whether different sizes of Por tumors affect rates of early recurrence and prognosis.

\section{Materials and Methods}

\section{Patients}

We identified 1,131 patients who underwent initial and curative hepatic resection for HCC without macroscopic vascular invasion at Osaka City University Hospital, Osaka, Japan between January 1991 and December 2018. Among them, 98 had preoperative transcatheter arterial embolization (TACE), and 24 were pathologically diagnosed with complete necrosis caused by preoperative TACE. Moreover, 24 patients were excluded from the study. Thus, a total of 1,107 patients were enrolled in the study. Cure affected by hepatic resection was defined as the histological absence of tu- mor cells along the liver transection plane. This study was conducted in accordance with the guidelines of the Ethics Committee of our institution (No. 3815) and the Declaration of Helsinki.

\section{Surgical Treatment}

The surgical indication was based on an algorithm that included the presence/absence of ascites, the serum total bilirubin level, and the results of the indocyanine green retention test [16]. For anatomic resection, we divided the liver parenchyma along the demarcation line that appeared on the liver surface after the occlusion of the portal vein and hepatic artery or after the injection of indocyanine green into the portal vein to outline the tumor-bearing area.

\section{Patient Follow-Up}

HCC-specific tumor markers were estimated, and ultrasonography, dynamic computed tomography $(\mathrm{CT})$, or magnetic resonance imaging was performed in every quarter after surgery. When imaging findings and/or an increase in tumor marker level indicated HCC recurrence, the patient underwent hepatic resection, radiofrequency ablation, TACE, or other alternative treatments, as indicated.

\section{Histological Study}

Histological examination was performed by experienced pathologists. The guidelines of the Liver Cancer Study Group of Japan [17] were used to evaluate the histological classifications of the tumor and the status of the background liver tissue. The tumor was graded histologically as well-differentiated, moderately differentiated, and Por HCC. The grade (severity of active hepatitis) and stage (degree of hepatic fibrosis) of noncancerous hepatic tissue were determined by scoring based on the histologic activity index $[18,19]$.

\section{Statistical Analysis}

Patients' demographic and clinical characteristics were calculated as numbers (proportions) and medians (interquartile ranges) for categorical and continuous variables, respectively. The differences between the characteristics of the Por HCCs and those of the well- or moderately differentiated (W-M) HCCs were analyzed by the $\chi^{2}$ test and the Mann-Whitney $U$ test. We examined the effect of the tumor differentiation on overall survival (OS), recurrence-free survival (RFS), cumulative early recurrence rate, and cumulative early extrahepatic recurrence rate using univariable and multivariable Cox proportional hazard regression models and Kaplan-Meier cumulative event-free probability estimators. Because 2 years after surgery was the inflection point [20], we defined postoperative recurrence within 2 years as early recurrence. To estimate the effect based on tumor size, each subgroup, which comprised patients who had tumors measuring $<2, \geq 2$ and $<5$, and $\geq 5 \mathrm{~cm}$, underwent similar analyses. In these models, the proportional hazard assumption was not satisfied, indicating that the effect of tumor differentiation changed over time. Therefore, we assessed the hazard ratios (HRs) of tumor differentiation before and after the second-year follow-up. Furthermore, based on the effect modification of tumor differentiation according to tumor size, multivariable Cox proportional hazards regression analyses, which used cross-product terms between tumor differentiation according to tumor size as explanatory variables, were performed. In these models, we analyzed the 
Table 1. Patient characteristics

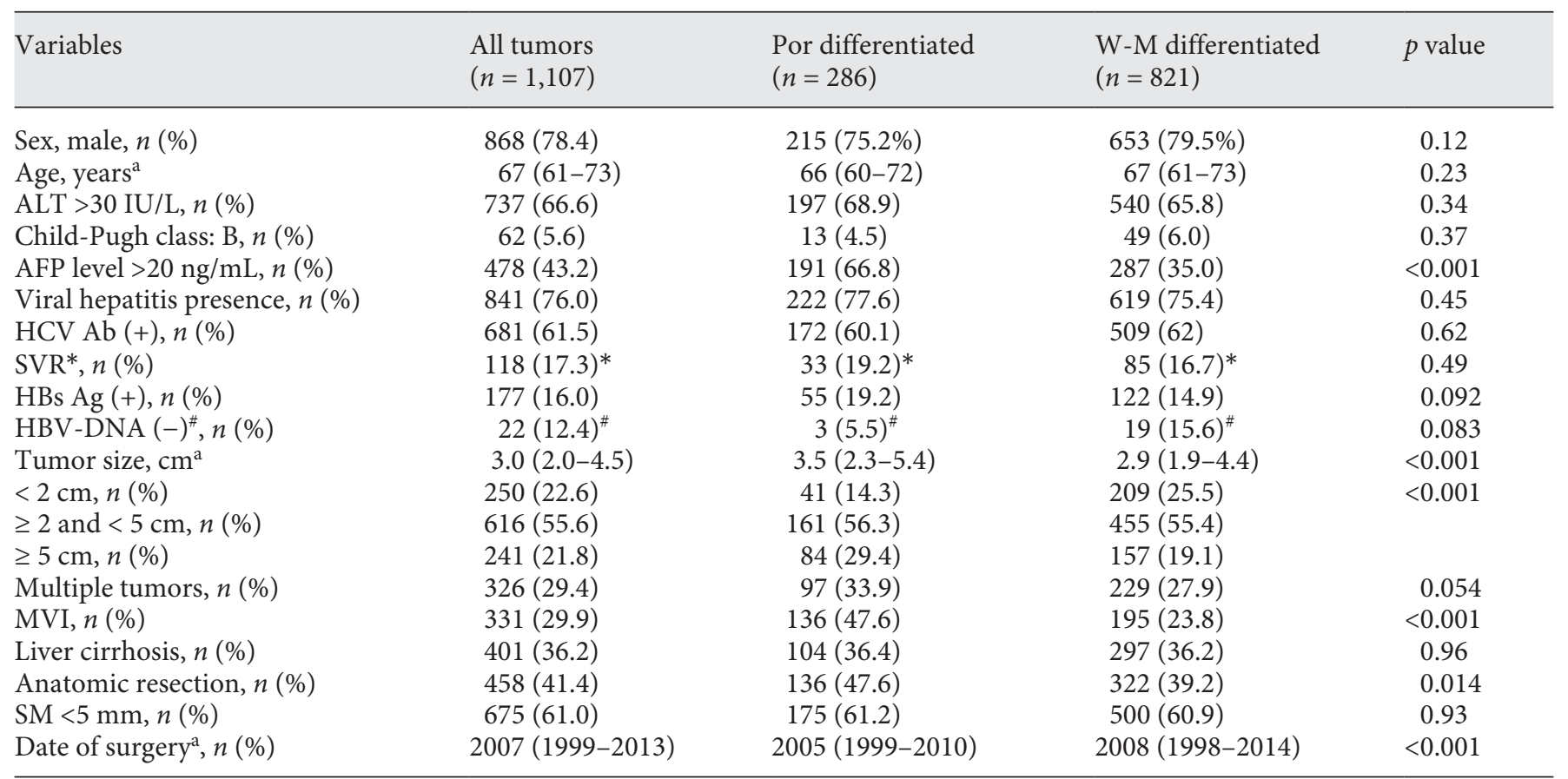

SVR was defined as a value for serum HCV-RNA under the detection sensitivity limit at 6 months after termination of interferon therapy or direct-acting antiviral therapy. HBV-DNA (-) was defined as the serum HBV-DNA concentration under the detection sensitivity limit at surgery by nulcleos(t)ide analogs. ALT, alanine aminotransferase; AFP, $\alpha$-fetoprotein; HCV Ab, anti-hepatitis C virus antibody; SVR, sustained virological response; HBV, hepatitis B virus; HBs Ag, hepatitis B surface antigen; MVI, microscopic vascular invasion; SM, surgical margin; Por, poorly differentiated; W-M, well- or moderately. ${ }^{a}$ Medians with interquartile ranges. * Ratio to patients with $\mathrm{HCV} \mathrm{Ab}(+) .{ }^{*}$ Ratio to patients with $\mathrm{HBs} \mathrm{Ag}(+)$.

nonlinear effect of the tumor size by using the restricted cubic spline method. Furthermore, these models were adjusted for the following covariables: age, sex, alanine aminotransferase level ( $\leq 30$ or $>30$ IU/L), Child-Pugh class (A or B), $\alpha$-fetoprotein level $(\leq 20$ or $>20 \mathrm{ng} / \mathrm{mL}$ ), presence of viral hepatitis (hepatitis B surface antigen positivity or anti-hepatitis $\mathrm{C}$ virus antibody positivity), multiple tumors, microscopic vascular invasion, liver cirrhosis, anatomic resection, surgical margin of $<5 \mathrm{~mm}$, and date of surgery. R software version 4.0.2 (https://www.r-project.org/) was used to perform all statistical hypothesis tests with 2 -sided $5 \%$ significance levels.

\section{Results}

\section{Clinicopathological Characteristics and Postoperative} Outcomes

Background characteristics of 286 patients with Por HCC and 821 with W-M HCC are listed in Table 1 . The proportions of patients with $\alpha$-fetoprotein levels $>20 \mathrm{ng} /$ $\mathrm{mL}$ and microvascular invasion were higher for Por
HCC than for W-M HCC. Patients with Por HCC exhibited more large tumors than those with W-M HCC. The median follow-up time was 49.5 (interquartile range 22.8-87.3) months. Postoperative recurrence was identified in 728 patients, and 568 patients died. Early recurrence and early extrahepatic recurrence were observed in 471 and 64 patients, respectively. The distribution of sites of early extrahepatic recurrence is described in online suppl. Table 1; for all online suppl. material, see www.karger.com/doi/10.1159/000517992.

\section{Survival Outcomes between Patients with $W-M$ and Those with Por HCCs}

Figure 1a-d show the Kaplan-Meier survival curves of OS, RFS, early RFS, and early extrahepatic RFS. The 5 -year OS and RFS rates were 64 and $30 \%$ in patients with W-M HCCs and 49 and $28 \%$ in patients with Por HCCs, respectively. The 2-year RFS rates of patients with $\mathrm{W}-\mathrm{M}$ HCCs and those with Por HCCs were 57 and 44\%, respectively. The 2-year extrahepatic RFS rates of the 2 


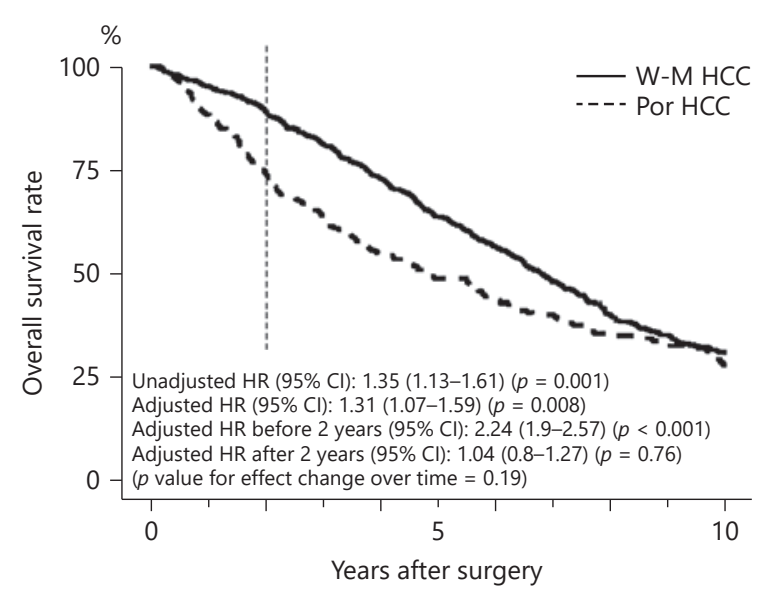

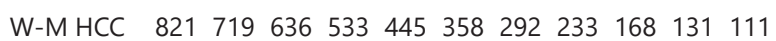
Por HCC $\begin{array}{llllllllllll}286 & 245 & 200 & 166 & 136 & 112 & 97 & 80 & 65 & 55 & 41\end{array}$

a

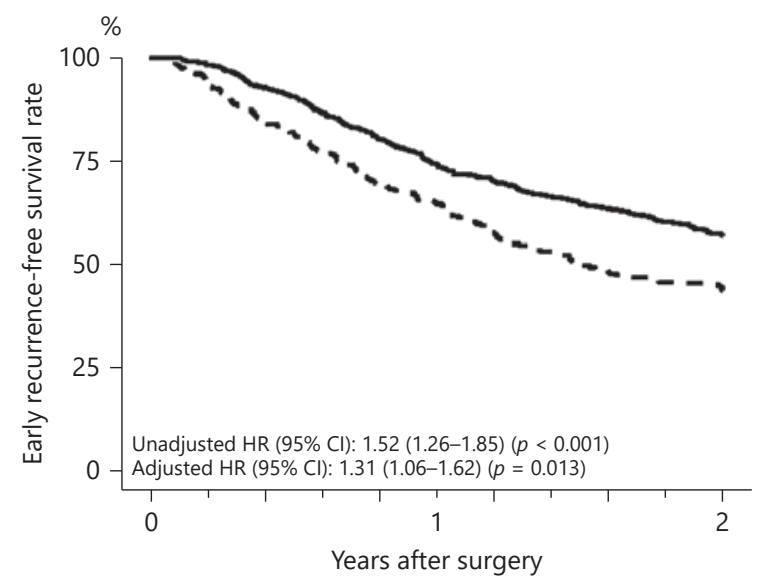

W-M HCC 821

Por HCC 286

$\begin{array}{ll}541 & 390 \\ 177 & 113\end{array}$

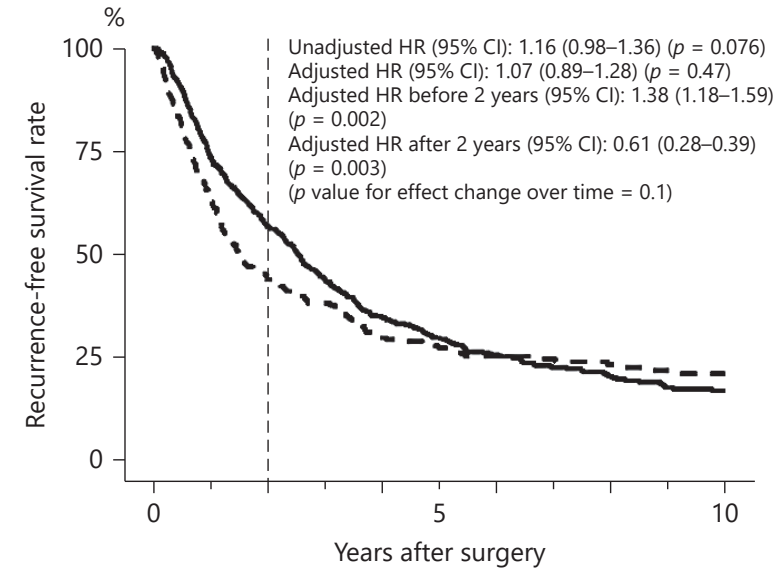

$\begin{array}{llllllllllll}\text { W-M HCC } & 821 & 541 & 390 & 267 & 191 & 140 & 107 & 84 & 63 & 47 & 43\end{array}$ Por HCC $\begin{array}{lllllllllll}286 & 177 & 113 & 89 & 66 & 54 & 45 & 39 & 35 & 28 & 22\end{array}$

b

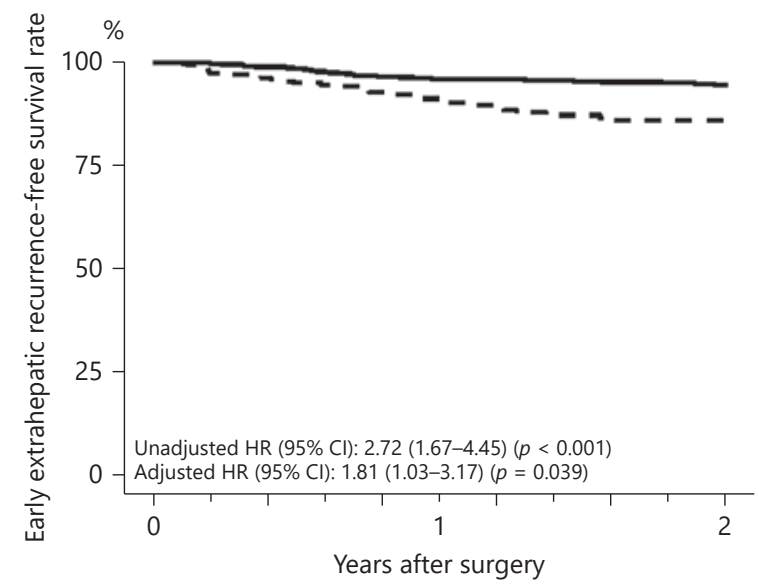

W-M HCC 821

Por HCC 286

541

177

390

d

ferentiation before and after the second-year follow-up were 2.24 and 1.04 ( $p$ value for effect change over time $=0.19$ ) for OS and 1.38 and 0.61 ( $p$ value for effect change over time $=0.1$ ) for RFS, respectively. Por, poorly differentiated; $\mathrm{W}-\mathrm{M}$, well- or moderately; HCC, hepatocellular carcinoma; OS, overall survival; RFS, recurrence-free survival; HRs, hazard ratios; $\mathrm{CI}$, confidence interval. groups were 95 and 86\%, respectively. The unadjusted Cox proportional hazards regression analysis revealed that the unadjusted HRs of reduced OS, RFS, early RFS, and early extrahepatic RFS in patients with Por HCCs were 1.35 (95\% confidence interval [CI] 1.13-1.61; $p=$ $0.001), 1.16$ (95\% CI 0.98-1.36; $p=0.076), 1.52$ (95\% CI $1.26-1.85 ; p<0.001)$, and 2.72 (95\% CI 1.67-4.45; $p<$
0.001), respectively. By multivariable Cox proportional hazards regression analysis, the adjusted HRs of reduced OS, RFS, early RFS, and early extrahepatic RFS in patients with Por HCCs were 1.31 (95\% CI 1.07-1.59; $p=$ $0.008), 1.07$ (95\% CI $0.89-1.28 ; p=0.47), 1.31(95 \% \mathrm{CI}$ $1.06-1.62 ; p=0.013)$, and $1.81(95 \%$ CI $1.03-3.17 ; p=$ $0.039)$, respectively. 


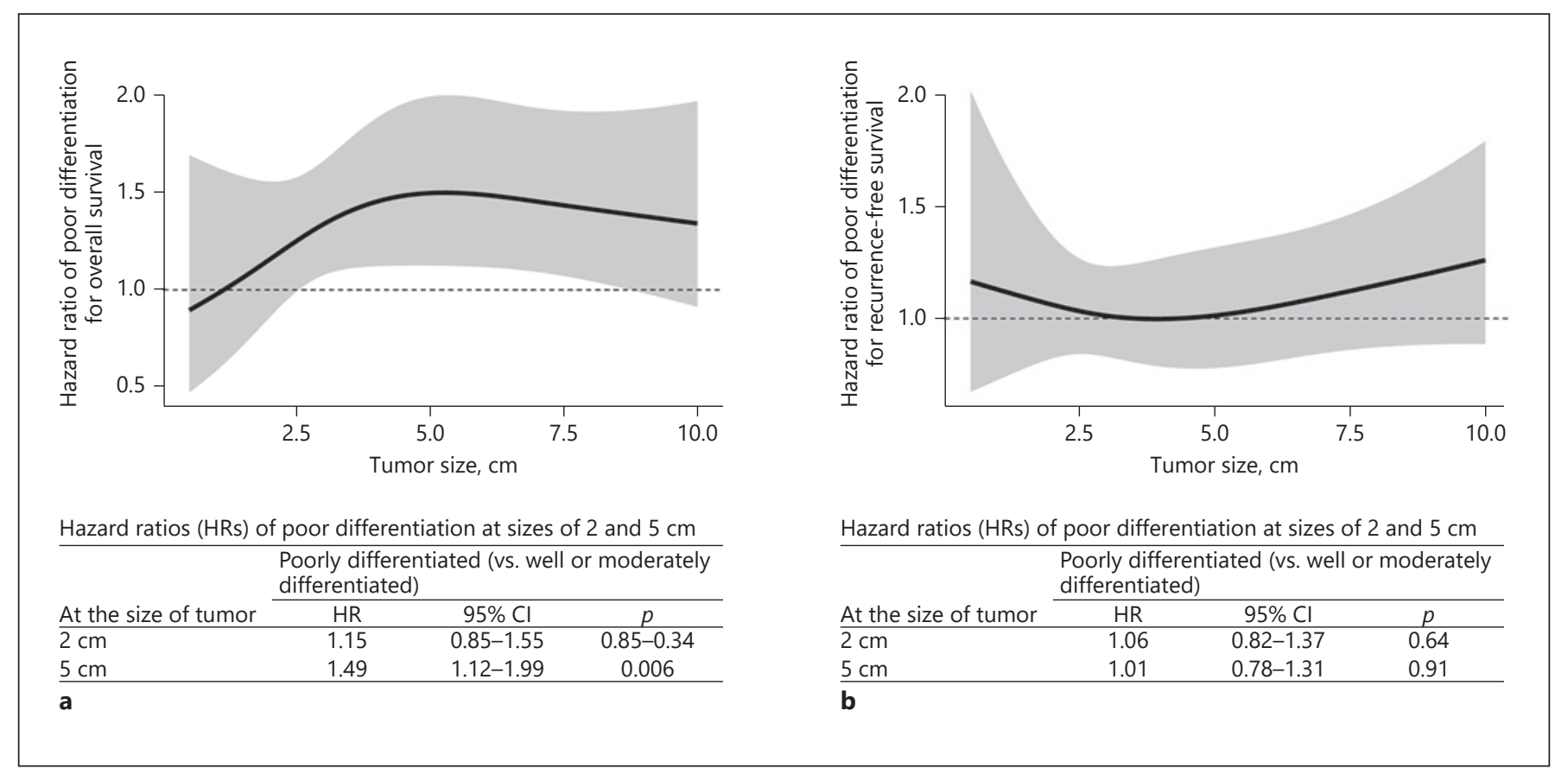

Fig. 2. The multivariable Cox proportional hazards regression models for assessing the effect modification of tumor differentiation according to tumor size in patients with Por differentiated HCC $(n=286)$ and W-M differentiated HCC $(n=821)$. Each HR and its $95 \%$ CI of poor differentiation in patients with tumors mea-

\section{Prognostic Effect of Tumor Differentiation on \\ Postoperative Outcomes with Regard to Changes in Tumor Size}

A multivariable Cox proportional hazards regression analysis was performed to assess effect modification. Results showed that Por HCC was associated with a lower OS $(p=0.033)$, but not with a reduced RFS $(p=0.54$; Fig. 2a, b). The HRs of Por HCCs for OS increased sharply until tumors reached around $5 \mathrm{~cm}$ in size. The HRs for reduction in OS were 1.49 (95\% CI 1.12-1.99; $p=0.006)$ for tumors $5 \mathrm{~cm}$ in size and 1.15 (95\% CI $0.85-1.55$; $p=$ 0.34 ) for tumors $2 \mathrm{~cm}$ in size. HR for OS did not change for the interaction between tumor differentiation and tumor size ( $\mathrm{p}$ for interaction $=0.45$ ).

Figure $3 \mathrm{a}, \mathrm{b}$ show the multivariable Cox proportional hazards regression model for early RFS and extrahepatic RFS. Results did not reveal a definite association between tumor differentiation and either early recurrence $(p=$ $0.07)$ or early extrahepatic recurrence $(p=0.15)$. However, patients with Por HCCs measuring about $5 \mathrm{~cm}$, but not those with tumors measuring approximately $2 \mathrm{~cm}$, were at higher risk for early recurrence (HR: 1.36, 1.19; 95\% CI: $1.00-1.84,0.85-1.65 ; p=0.048,0.29$, respective- suring 2 and $5 \mathrm{~cm}$. a OS. b RFS. Por, poorly differentiated; W-M, well- or moderately; HCC, hepatocellular carcinoma; OS, overall survival; RFS, recurrence-free survival; HRs, hazard ratios; CI, confidence interval.

ly) and early extrahepatic recurrence (HR: 2.26, 1.58; 95\% CI: $1.07-4.78,0.42-5.90 ; p=0.033,0.49$, respectively). The HRs of early recurrence and early extrahepatic recurrence did not change for the interaction between tumor differentiation and tumor size $(p$ for interaction $=0.64$ and 0.59 , respectively).

\section{Prognostic Effect of Tumor Differentiation on}

Postoperative Outcomes Stratified according to Tumor

Size

Figure $4 \mathrm{a}-\mathrm{d}$ show the Kaplan-Meier survival curves of OS, RFS, early RFS, and early extrahepatic RFS in patients with tumors measuring $<2, \geq 2$ and $<5$, and $\geq 5 \mathrm{~cm}$. The 5 -year OS rates of patients with W-M and Por HCCs measuring $<2, \geq 2$ and $<5$, and $\geq 5 \mathrm{~cm}$ were 68 and $57 \%, 63$ and $56 \%$, and 60 and $30 \%$, respectively. Their corresponding 5 -year RFS rates were 33 and 32\%, 28 and 29\%, 30 and $23 \%$, respectively. The 2-year RFS rates of patients with $\mathrm{W}$-M and Por HCCs measuring $<2, \geq 2$ and $<5$, and $\geq 5 \mathrm{~cm}$ were 65 and 59\%, 56 and 47\%, and 48 and 30\%, respectively. Their corresponding 5 -year early extrahepatic RFS rates were 99 and 98\%, 95 and $90 \%$, and 86 and 70\%, respectively. The unadjusted Cox proportional hazards re- 


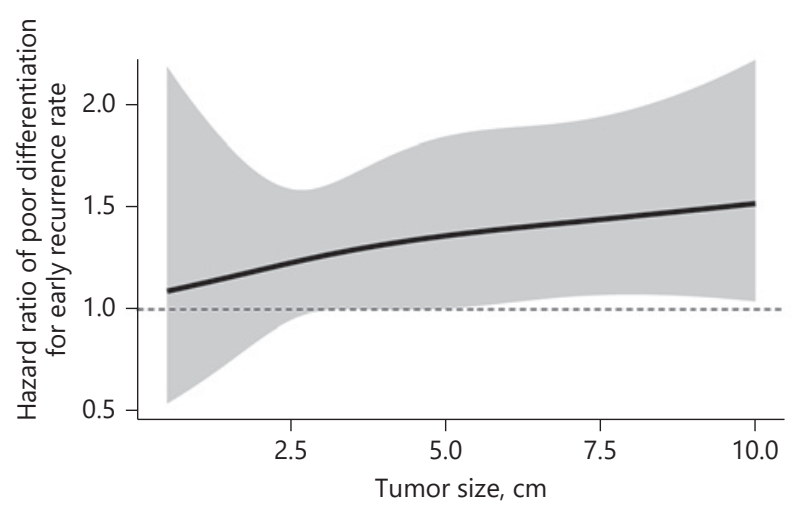

Hazard ratios (HRs) of poor differentiation at sizes of 2 and $5 \mathrm{~cm}$

\begin{tabular}{lccc}
\hline & \multicolumn{3}{l}{$\begin{array}{l}\text { Poorly differentiated (vs. well or moderately } \\
\text { differentiated) }\end{array}$} \\
\cline { 2 - 4 } At the size of tumor & $\mathrm{HR}$ & $95 \% \mathrm{Cl}$ & $p$ \\
\hline $2 \mathrm{~cm}$ & 1.19 & $0.85-1.65$ & 0.29 \\
$5 \mathrm{~cm}$ & 1.36 & $1.00-1.84$ & 0.048 \\
\hline
\end{tabular}

a

Fig. 3. The multivariable Cox proportional hazards regression models for assessing the effect modification of tumor differentiation according to tumor size for early recurrence (within 2 years after surgery) of Por differentiated HCC $(n=286)$ and W-M differentiated HCC $(n=821)$. Each HR and its $95 \%$ CI of poor dif-

gression analysis showed that the unadjusted HRs of reduced OS in patients with Por HCCs measuring $<2, \geq 2$ and $<5$, and $\geq 5 \mathrm{~cm}$ were 1.16 (95\% CI $0.71-1.89 ; p=0.55)$, 1.14 (95\% CI $0.9-1.45 ; p=0.28$ ), and 1.77 (95\% CI $1.27-$ 2.48; $p<0.001)$, respectively. The corresponding unadjusted HRs of reduced RFS were 0.92 (95\% CI 0.59-1.43; $p=0.70), 1.05$ (95\% CI 0.85-1.3; $p=0.65)$, and 1.48 (95\% CI 1.07-2.04; $p=0.016)$, respectively. The corresponding unadjusted HRs of reduced early RFS were 1.18 (95\% CI $0.67-2.07 ; p=0.56$ ), 1.3 (95\% CI $1-1.69 ; p=0.047)$, and 1.86 (95\% CI 1.31-2.63; $p<0.001)$, respectively. The corresponding unadjusted HRs of reduced early extrahepatic RFS were 5.22 (95\% CI 0.33-83.51; $p=0.24), 2.27$ (95\% CI $1.09-4.72 ; p=0.028)$, and $2.19(95 \%$ CI $1.11-4.35 ; p=$ $0.024)$, respectively.

Based on a multivariable Cox proportional hazards regression analysis, the adjusted HRs of reduced OS in patients with Por HCCs measuring $<2, \geq 2$ and $<5$, and $\geq 5 \mathrm{~cm}$ were 1.22 (95\% CI $0.69-2.14 ; p=0.50), 1.33$ (95\% CI 1.02$1.73 ; p=0.034)$, and 1.58 (95\% CI 1.04-2.42; $p=0.033$ ), respectively. The corresponding adjusted HRs of reduced RFS were 0.8 (95\% CI 0.49-1.33; $p=0.40), 1.08$ (95\% CI $0.86-1.37 ; p=0.50)$, and 1.22 (95\% CI $0.83-1.81 ; p=0.32)$,

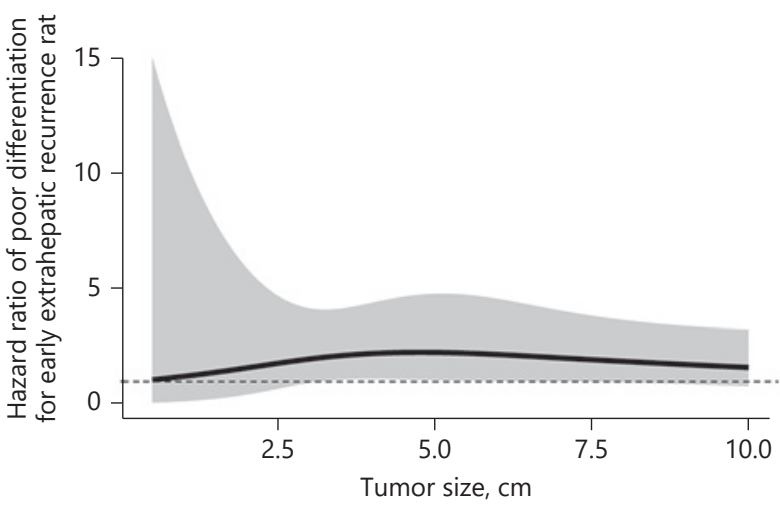

\begin{tabular}{lccc} 
Hazard ratios (HRs) of poor differentiation at sizes of 2 and $5 \mathrm{~cm}$ \\
\hline \multicolumn{4}{c}{$\begin{array}{l}\text { Poorly differentiated (vs. well or moderately } \\
\text { differentiated) }\end{array}$} \\
\cline { 2 - 4 } At the size of tumor & $\mathrm{HR}$ & $95 \% \mathrm{Cl}$ & $p$ \\
\hline $2 \mathrm{~cm}$ & 1.58 & $0.42-5.90$ & 0.49 \\
$5 \mathrm{~cm}$ & 2.26 & $1.07-4.78$ & 0.033 \\
\hline
\end{tabular}

b ferentiation for tumors measuring 2 and $5 \mathrm{~cm}$. Por, poorly differentiated; W-M, well- or moderately; HCC, hepatocellular carcinoma; HRs, hazard ratios; CI, confidence interval; OS, overall survival; RFS, recurrence-free survival.

respectively. The corresponding adjusted HRs of reduced early RFS were 0.85 (95\% CI $0.46-1.57 ; p=0.60), 1.34$ (95\% CI 1.01-1.8; $p=0.045$ ), and 1.57 (95\% CI 1.03-2.39; $p=0.036$ ), respectively. The adjusted HRs of reduced early extrahepatic RFS in patients with tumors measuring $\geq 2$ and $<5$ and $\geq 5 \mathrm{~cm}$ were 1.89 (95\% CI $0.83-4.3 ; p=0.13$ ) and 2.33 (95\% CI 0.98-5.54; $p=0.056)$, respectively. However, the adjusted HR of tumors measuring $<2 \mathrm{~cm}$ could not be estimated due to the small number of events.

\section{Discussion}

Multivariable Cox proportional hazards regression analysis revealed that Por HCC was associated with reduced OS, early RFS, and early extrahepatic RFS. However, its correlation with RFS was not confirmed. By assessing the effect modification of tumor differentiation according to tumor size, Por HCC was found to be associated with reduced OS, and the HRs of Por HCCs sharply increased in patients with tumors measuring up to $5 \mathrm{~cm}$. Patients with Por HCCs measuring about $5 \mathrm{~cm}$ were at risk for low OS. Nevertheless, the risk was not confirmed 


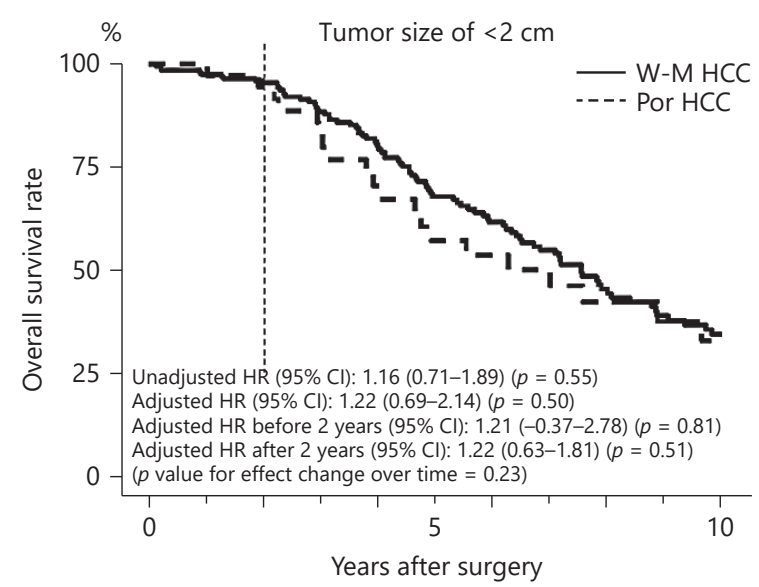

$\begin{array}{llllllllllll}\text { W-M HCC } & 209 & 189 & 176 & 143 & 118 & 90 & 77 & 65 & 44 & 35 & 30\end{array}$ $\begin{array}{lllllllllllll}\text { Por HCC } & 41 & 36 & 34 & 28 & 22 & 17 & 15 & 13 & 10 & 8 & 7\end{array}$

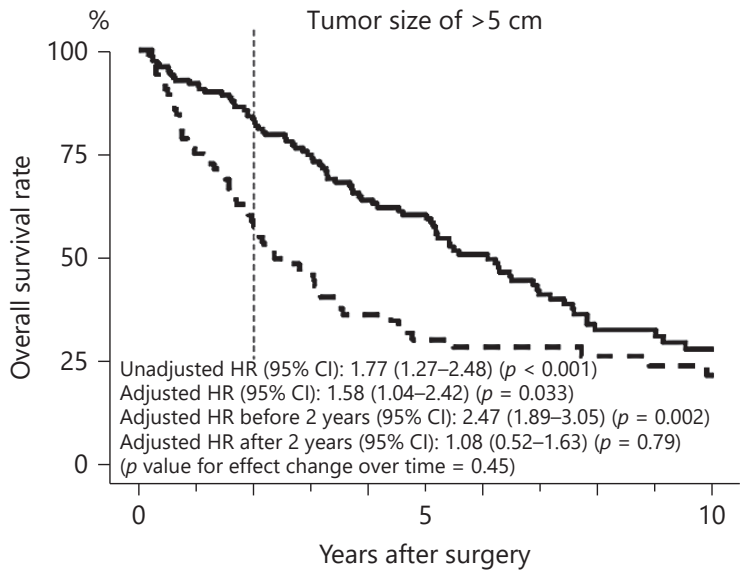

$\begin{array}{llllllllllll}\text { W-M HCC } & 157 & 133 & 111 & 92 & 72 & 65 & 49 & 37 & 25 & 21 & 18\end{array}$

$\begin{array}{llllllllllll}\text { Por HCC } & 84 & 62 & 44 & 34 & 26 & 17 & 16 & 14 & 12 & 10 & 9\end{array}$

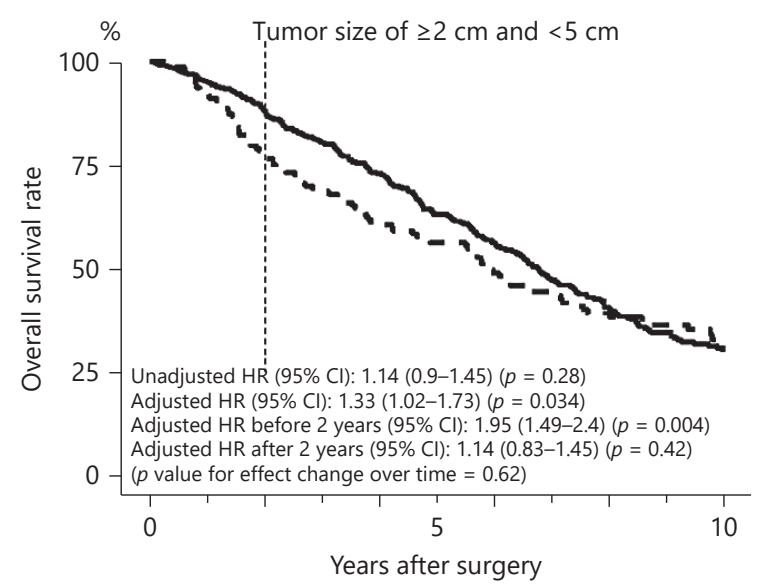

W-M HCC $4 \begin{array}{lllllllllll}455 & 397 & 349 & 298 & 255 & 203 & 166 & 161 & 99 & 75 & 63\end{array}$

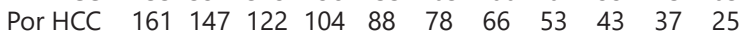

a

Fig. 4. Kaplan-Meier survival curves between patients with W-M differentiated HCC and Por differentiated HCC measuring $<2, \geq 2$ and $<5$, and $\geq 5 \mathrm{~cm}$. a OS. b RFS. c Early RFS. d Early extrahepatic RFS. The unadjusted and adjusted HRs of reduced OS, RFS, early RFS, and early extrhepatic RFS in patients with Por HCCs were calculated using unadjusted and adjusted Cox proportional hazard regression models. The HRs of OS in patients with tumor differentiation before and after the second-year follow-up were 1.21 and 1.22 ( $p$ value for effect change over time $=0.23$ ) for tumors measuring $<2 \mathrm{~cm}, 1.95$ and 1.14 ( $p$ value for effect change over time $=$

in patients with tumors measuring about $2 \mathrm{~cm}$. Similarly, patients with Por HCCs measuring approximately $5 \mathrm{~cm}$, but not those with tumors measuring about $2 \mathrm{~cm}$, had a higher risk for early and extrahepatic recurrence. Furthermore, by multivariable Cox proportional hazards re-
0.62 ) for tumors measuring $\geq 2$ and $<5 \mathrm{~cm}$, and 2.47 and 1.08 ( $p$ value for effect change over time $=0.45$ ) for tumors measuring $\geq 5 \mathrm{~cm}$, respectively. The corresponding HRs of RFS were 0.95 and 0.61 ( $p$ value for effect change over time $=0.94), 1.34$ and 0.74 ( $p$ value for effect change over time $=0.84$ ), and 1.5 and 0.37 ( $p$ value for effect change over time $=0.038$ ), respectively. Por, poorly differentiated; W-M, well- or moderately; HCC, hepatocellular carcinoma; OS, overall survival; RFS, recurrence-free survival; HRs, hazard ratios; $\mathrm{CI}$, confidence interval.

(Figure continued on next pages.)

gression analysis stratified according to tumor size, patients with Por HCCs measuring $\geq 2$ and $<5$ and $\geq 5 \mathrm{~cm}$ had a higher risk of low OS and early recurrence. In addition, Por HCCs measuring $\geq 5 \mathrm{~cm}$ was correlated with a higher risk of early extrahepatic recurrence. 

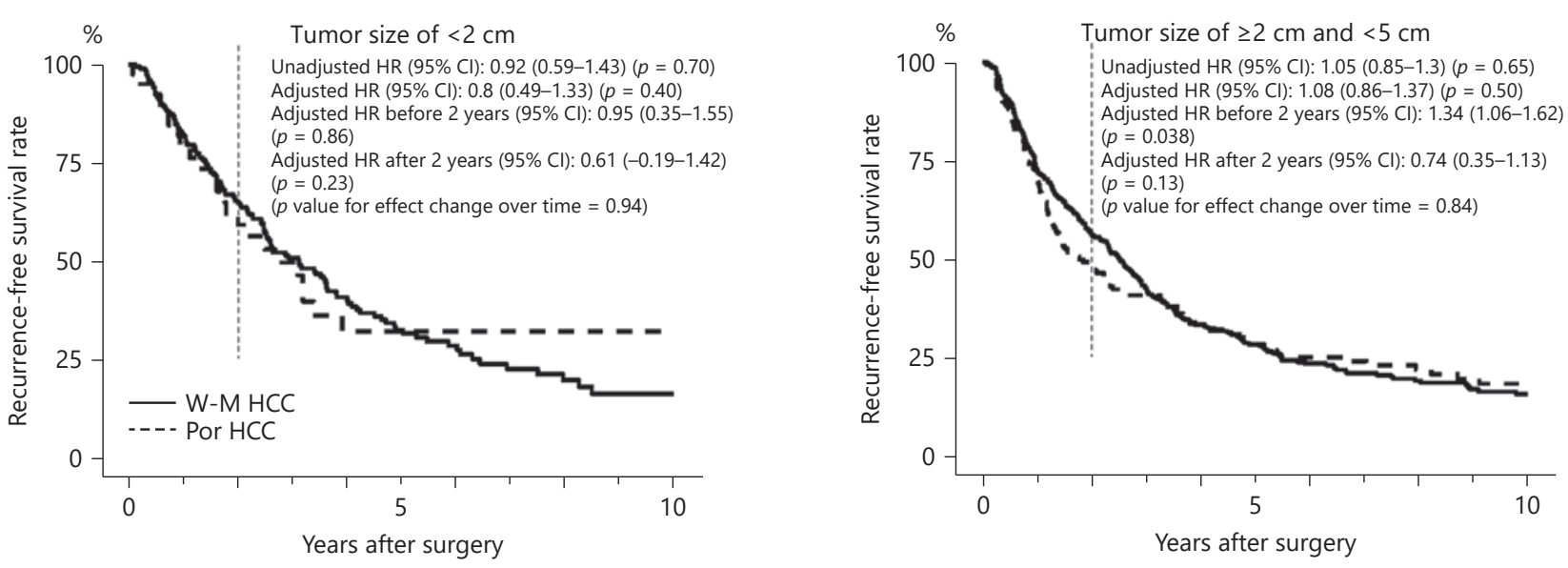
$\begin{array}{rccccccccccc}\text { W-M HCC } & 209 & 156 & 117 & 76 & 52 & 34 & 26 & 18 & 13 & 9 & 9 \\ \text { Por HCC } & 41 & 28 & 21 & 15 & 8 & 8 & 8 & 7 & 6 & 5 & 5\end{array}$

$\begin{array}{llllllllllll}\text { W-M HCC } & 455 & 293 & 214 & 148 & 108 & 79 & 59 & 50 & 38 & 29 & 25\end{array}$
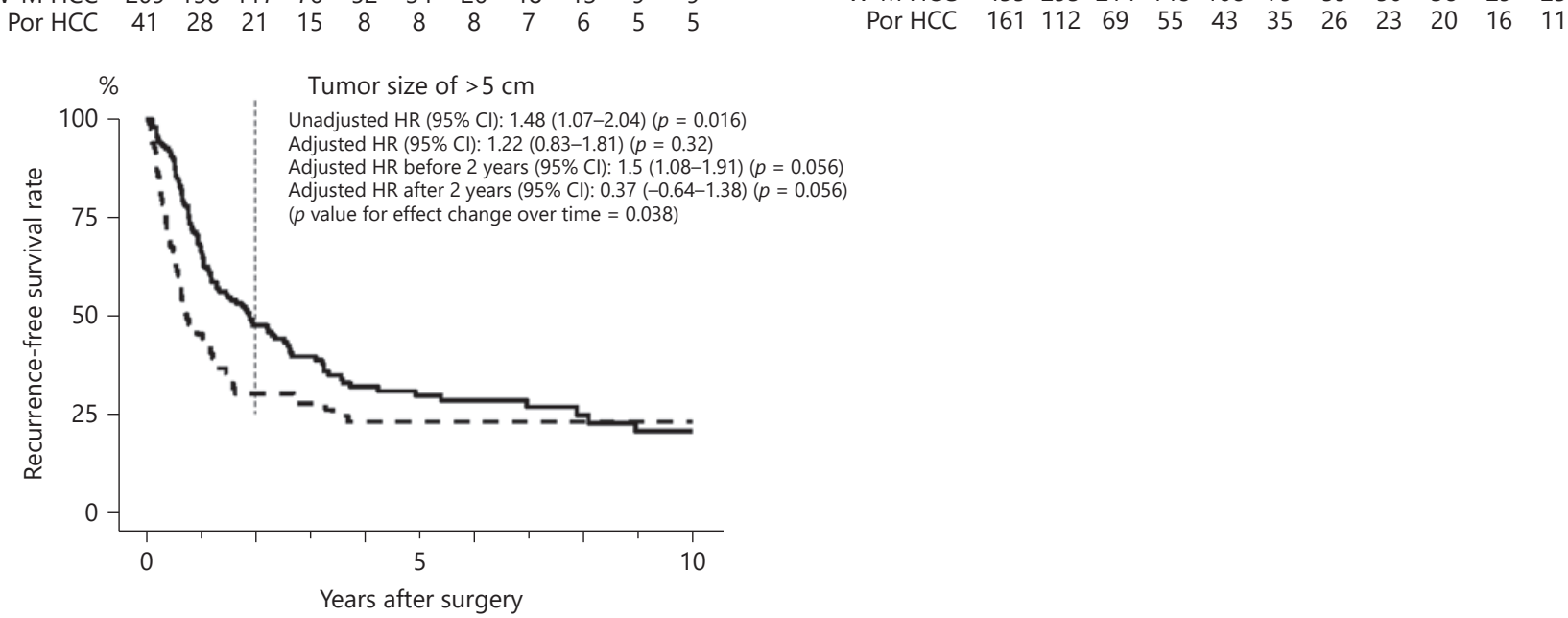

$\begin{array}{llllllllllll}\text { W-M HCC } & 157 & 92 & 59 & 43 & 31 & 27 & 22 & 16 & 12 & 9 & 9\end{array}$

$\begin{array}{llllllllllll}\text { Por HCC } & 84 & 37 & 23 & 19 & 15 & 11 & 11 & 9 & 9 & 7 & 6\end{array}$

b

4

Tumor differentiation has been shown by many studies to have no significant effect on OS after surgery [1113]. One possible reason for this might be the influence of tumor size. In our study, the HRs of Por HCCs sharply increased in patients with tumors measuring up to $5 \mathrm{~cm}$. Furthermore, patients with Por HCCs measuring $\geq 2$ and $<5$ and $\geq 5 \mathrm{~cm}$, but not those with tumors measuring $<2$ $\mathrm{cm}$, had a higher risk. Therefore, the proportion of small HCCs may have influenced the risk of Por HCC for OS in prior studies.

Poor differentiation of HCCs has been reported to be associated with tumor aggressiveness, including progression, invasion, and metastasis $[9,10,21,22]$. Endo and
Terada [10] reported that upregulation of CD44 isoform, a transmembrane glycoprotein that acts mainly as a receptor for hyaluronan to function in cell adhesion, was associated with vascular invasion and Por HCC. The reduced expression of E-cadherin, a cell adhesion factor, was reported to be associated with tumor progression and Por HCC [22]. Furthermore, as tumor size increased, Por HCC was demonstrated to increase the incidence of microscopic vascular invasion [14] and the frequency of extrahepatic recurrence after surgery [11]. These findings indicated that the aggressive behavior of Por HCC might become evident as it enlarges. In our study, Por HCCs measuring $\geq 2$ and $<5$ and $\geq 5 \mathrm{~cm}$ were associated with a 


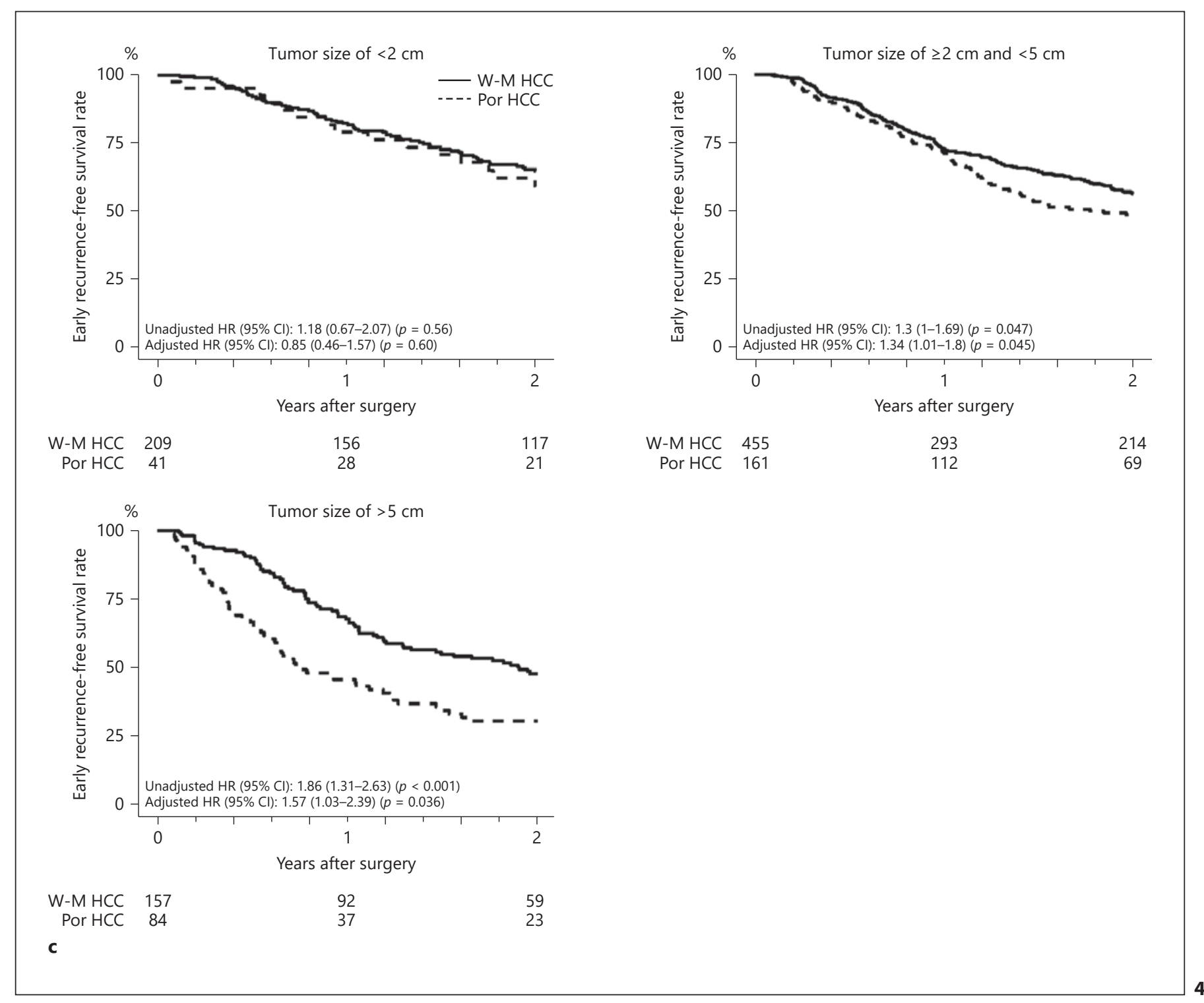

high risk of early recurrence, and Por HCCs measuring $\geq 5 \mathrm{~cm}$ were correlated with a high risk of early extrahepatic recurrence.

Early recurrence (within 2 years of surgery) is considered to arise from residual micrometastasis, including intrahepatic and extrahepatic portion [20]. In our study, patients with Por HCCs measuring $5 \mathrm{~cm}$, but not those with tumors measuring $2 \mathrm{~cm}$, had a high risk of early recurrence. Moreover, patients with Por HCCs measuring $\geq 2$ and $<5 \mathrm{~cm}$ and those with tumors measuring $\geq 5 \mathrm{~cm}$ were at risk for early recurrence. Furthermore, Por HCCs measuring $\geq 5 \mathrm{~cm}$ were correlated with a higher risk for early extrahepatic recurrence. Therefore, patients with
Por HCCs measuring $\geq 2 \mathrm{~cm}$ were more likely to develop hematogenous micrometastasis. Meanwhile, the rate of long-term survival of patients with early recurrence or extrahepatic recurrence is very poor [23]. In our study, the prognostic risk for reduced OS was evident in patients with Por HCCs measuring $\geq 2$ and $<5 \mathrm{~cm}$ and those with tumors measuring $\geq 5 \mathrm{~cm}$. In view of the causal relationship between early recurrence and hematogenous micrometastasis, early recurrence of Por HCC may recur early via hematogenous spread, and thus OS may be reduced in patients with tumors measuring $\geq 2 \mathrm{~cm}$.

Meanwhile, patients with Por HCCs measuring $<2 \mathrm{~cm}$ did not have a high risk for early recurrence, early extra- 


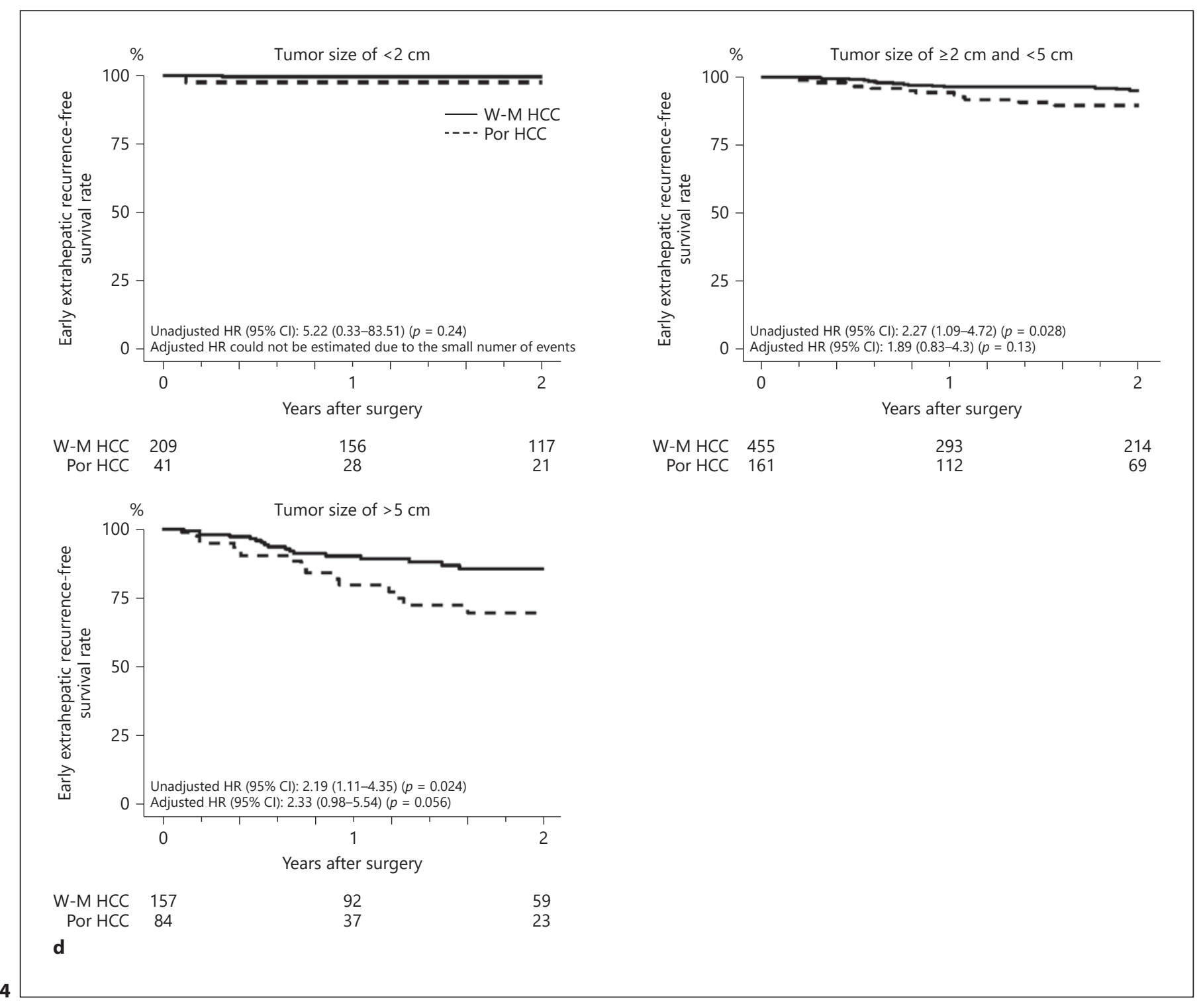

hepatic recurrence, or low OS in our study. Previous studies indicated excellent long-term outcomes in patients with HCCs up to $2 \mathrm{~cm}$ in size [24]. The cutoff size of $2 \mathrm{~cm}$ has been adopted in the Barcelona Clinic Liver Cancer staging system, and HCCs $\leq 2 \mathrm{~cm}$ in size are categorized as "very early HCC" [5]. The prognosis of patients with HCCs measuring $\leq 2 \mathrm{~cm}$ is considered not to be affected by the presence of microscopic vascular invasion [15]. In our study, patients with Por and W-M HCCs measuring $<2 \mathrm{~cm}$ had similar Kaplan-Meier survival curves of early RFS and early extrahepatic RFS. Thus, in patients with small HCCs measuring up to $2 \mathrm{~cm}$, tumor differentiation was not associated with early recurrence or early extrahe- patic recurrence, and it had no effect on long-term survival.

Our results raise clinically important concerns. First, clinicians can facilitate plans for individual case management after hepatic resection for Por HCCs according to tumor size. Due to the increased risk of early recurrence in patients with Por HCCs measuring $\geq 2 \mathrm{~cm}$, physicians should increase the frequency of patient visits. In patients with Por HCCs measuring $\geq 5 \mathrm{~cm}$, considering the increased risk of early extrahepatic recurrence, physicians should perform an extrahepatic metastasis workup, including chest CT scan, brain CT scan, and whole-body bone scintigraphy in addition to routine abdominal im- 
aging follow-up. Second, among patients with Por HCC, it may be possible to determine potential candidates for adjuvant therapy. Although no effective therapies currently exist in adjuvant settings, several immune checkpoint inhibitors, including nivolumab and pembrolizum$\mathrm{ab}$, have recently demonstrated durable response effects for patients with advanced HCC [25]. Many trials of immune checkpoint inhibitors are ongoing in the adjuvant setting [26]. To select candidates for adjuvant therapy in clinical trials, several criteria have been devised on the basis of tumor progression $[27,28]$. Considering the increased risk for early recurrence, early extrahepatic recurrence, and reduced OS, Por HCC measuring $\geq 5 \mathrm{~cm}$ could be an additional criterion for adjuvant chemotherapy after surgery. Third, various curative therapeutic options are available for HCCs measuring $<2 \mathrm{~cm}$, which includes hepatic resection, local ablation therapy, or liver transplantation according to tumor number and background liver function because Por HCCs measuring $<2 \mathrm{~cm}$ are not associated with the risk of early recurrence and poor OS.

Meanwhile, Por HCC was not associated with an increased risk for reduced RFS in the multivariable Cox proportional hazards regression model, whereas it was associated with low OS. One possible reason for the discrepancy is the influence of multicentric recurrence. Multicentric recurrence is considered to be de novo HCC in the remnant liver, typically manifesting $>2$ years after surgery [29]. Patients with multicentric recurrence have a chance to undergo curative treatment and have a favorable prognosis [30]. In this study, the study period was 27 years, and cases of multicentric recurrence would have accumulated during this time. In fact, patients with Por HCCs measuring $\geq 2 \mathrm{~cm}$ had a risk for early recurrence in this study. Therefore, multicentric recurrence might obscure the risk of Por HCC for reduced RFS.

This study had several limitations. First, the retrospective nature of the study may have resulted in some bias in patient enrollment. Second, patients who preoperatively underwent TACE might have tumor specimens with necrotic components in part. Therefore, the diagnostic limitation of tumor differentiation associated with TACE could have existed. However, in total, only 74 patients underwent preoperative TACE. Thus, the influence of the diagnostic limitation of tumor differentiation on survival could be quite limited. Third, our results may have been influenced by the long study period, inasmuch as medical techniques and treatment selection have changed. However, we adjusted the confounding effect of the period of surgery by entering it into multivariable Cox proportional hazards regression models. Furthermore, the current results of the long-term study period could provide important information for the treatment of HCC.

\section{Conclusion}

In conclusion, Por HCC measuring $\geq 2 \mathrm{~cm}$ was associated with early recurrence. Hence, it had a negative effect on OS. After surgery, patients with Por HCC measuring $\geq 5 \mathrm{~cm}$ should be cautiously assessed for early extrahepatic recurrence. These findings will help physicians devise treatment strategies for patients with HCC.

\section{Acknowledgments}

This work was supported by the Health, Labor, and Welfare Policy Research Grants from the Ministry of Health, Labor, and Welfare of Japan (Policy Research for Hepatitis Measures [H30Kansei-Shitei-003]).

\section{Statement of Ethics}

The participants provided a written informed consent, and the study protocol was approved by the human research committee of the institution (No. 3815). The research was conducted ethically in accordance with the https://www.wma.net/policies-post/wmadeclaration-of-helsinki-ethical-principles-for-medical-researchinvolving-human-subjects/. World Medical Association Declaration of Helsinki.

\section{Conflict of Interest Statement}

The authors have no conflicts of interest to declare.

\section{Funding Sources}

This work was supported by the Health, Labor, and Welfare Policy Research Grants from the Ministry of Health, Labor, and Welfare of Japan (Policy Research for Hepatitis Measures [H30Kansei-Shitei-003]).

\section{Author Contributions}

Conception and design were contributed by Shinkawa and Kubo. Data acquisition and analysis were contributed by Tanaka, Takemura, Kinoshita, Amano, and Kinoshita. Data analysis and 
interpretation was contributed by Shinkawa, Tanaka, Takemura, Kimura, and Kabata. Drafting of the manuscript was contributed by Shinkawa. Critical revision of the manuscript was contributed by Kubo. Final approval was given by all authors who approved the final version of the manuscript.

\section{Data Availability Statement}

All data generated or analyzed during this study are included in this article and its online suppl. Material files. Further inquiries can be directed to the corresponding author.

\section{References}

1 Bruix J, Gores GJ, Mazzaferro V. Hepatocellular carcinoma: clinical frontiers and perspectives. Gut. 2014 May;63(5):844-55.

2 Kluger MD, Salceda JA, Laurent A, Tayar C, Duvoux C, Decaens T, et al. Liver resection for hepatocellular carcinoma in 313 Western patients: tumor biology and underlying liver rather than tumor size drive prognosis. J Hepatol. 2015 May;62(5):1131-40.

3 Kubo S, Tanaka H, Shuto T, Takemura S, Yamamoto T, Uenishi T, et al. Prognostic effects of causative virus in hepatocellular carcinoma according to the Japan integrated staging (JIS) score. J Gastroenterol. 2005 Oct;40(10):972-9.

4 Koda M, Tanaka S, Takemura S, Shinkawa $\mathrm{H}$, Kinoshita M, Hamano G, et al. Long-term prognostic factors after hepatic resection for hepatitis $C$ virus-related hepatocellular carcinoma, with a special reference to viral status. Liver Cancer. 2018 Sep;7(3):261-76.

5 European Association For The Study Of The Liver. EASL clinical practice guidelines: management of hepatocellular carcinoma. J Hepatol. 2018 Jul;69(1):182-236.

6 Kubo S, Hirohashi K, Tanaka H, Shuto T, Takemura S, Yamamoto T, et al. Usefulness of viral concentration measurement by transcription-mediated amplification and hybridization protection as a prognostic factor for recurrence after resection of hepatitis $B$ virusrelated hepatocellular carcinoma. Hepatol Res. 2003 Jan;25(1):71-7.

7 Shinkawa H, Tanaka S, Takemura S, Ishihara T, Yamamoto K, Kubo S. Tumor size drives the prognosis after hepatic resection of solitary hepatocellular carcinoma without vascular invasion. J Gastrointest Surg. 2020 May; 24(5):1040-8.

8 Karadag Soylu N. Update on hepatocellular carcinoma: a brief review from pathologist standpoint. J Gastrointest Cancer. 2020 Dec; 51(4):1176-86

9 Lee H, Ryu SH, Hong SS, Seo DD, Min HJ, Jang MK, et al. Overexpression of metastasisassociated protein 2 is associated with hepatocellular carcinoma size and differentiation. J Gastroenterol Hepatol. 2009 Aug;24(8): 1445-50.

10 Endo K, Terada T. Protein expression of CD44 (standard and variant isoforms) in hepatocellular carcinoma: relationships with tumor grade, clinicopathologic parameters, p53 expression, and patient survival. J Hepatol. 2000 Jan;32(1):78-84.
11 Oishi K, Itamoto T, Amano H, Fukuda S, Ohdan H, Tashiro H, et al. Clinicopathologic features of poorly differentiated hepatocellular carcinoma. J Surg Oncol. 2007 Mar;95(4): 311-6.

12 Lim C, Mise Y, Sakamoto Y, Yamamoto S, Shindoh J, Ishizawa $\mathrm{T}$, et al. Above $5 \mathrm{~cm}$, size does not matter anymore in patients with hepatocellular carcinoma. World J Surg. 2014 Nov;38(11):2910-8.

13 Zhao H, Hua Y, Lu Z, Gu S, Zhu L, Ji Y, et al. Prognostic value and preoperative predictors of microvascular invasion in solitary hepatocellular carcinoma $\leq 5 \mathrm{~cm}$ without macrovascular invasion. Oncotarget. 2017 May;8(37): 61203-14.

14 Pawlik TM, Delman KA, Vauthey JN, Nagorney DM, Ng IO, Ikai I, et al. Tumor size predicts vascular invasion and histologic grade: implications for selection of surgical treatment for hepatocellular carcinoma. Liver Transpl. 2005 Sep;11(9):1086-92.

15 Shindoh J, Andreou A, Aloia TA, Zimmitti G, Lauwers GY, Laurent A, et al. Microvascular invasion does not predict long-term survival in hepatocellular carcinoma up to $2 \mathrm{~cm}$ : reappraisal of the staging system for solitary tumors. Ann Surg Oncol. 2013 Apr;20(4):1223-9.

16 Makuuchi M, Kosuge T, Takayama T, Yamazaki S, Kakazu T, Miyagawa S, et al. Surgery for small liver cancers. Semin Surg Oncol. 1993 Jul-Aug;9(4):298-304.

17 The general rules for the clinical and pathological study of primary liver cancer. Liver Cancer Study Group of Japan. Jpn J Surg. 1989 Jan;19(1):98-129.

18 Knodell RG, Ishak KG, Black WC, Chen TS, Craig R, Kaplowitz N, et al. Formulation and application of a numerical scoring system for assessing histological activity in asymptomatic chronic active hepatitis. Hepatology. 1981 Apr;1(5):431-5.

19 Desmet VJ, Gerber M, Hoofnagle JH, Manns $\mathrm{M}$, Scheuer PJ. Classification of chronic hepatitis: diagnosis, grading and staging. Hepatology. 1994 Jun;19(6):1513-20.

20 Portolani N, Coniglio A, Ghidoni S, Giovanelli M, Benetti A, Tiberio GA, et al. Early and late recurrence after liver resection for hepatocellular carcinoma: prognostic and therapeutic implications. Ann Surg. 2006 Feb; 243(2):229-35.
21 Hou KZ, Fu ZQ, Gong H. Chemokine ligand 20 enhances progression of hepatocellular carcinoma via epithelial-mesenchymal transition. World J Gastroenterol. 2015 Jan;21(2): 475-83.

22 Endo K, Ueda T, Ueyama J, Ohta T, Terada T. Immunoreactive E-cadherin, alpha-catenin, beta-catenin, and gamma-catenin proteins in hepatocellular carcinoma: relationships with tumor grade, clinicopathologic parameters, and patients' survival. Hum Pathol. 2000 May;31(5):558-65.

23 Taketomi A, Toshima T, Kitagawa D, Motomura T, Takeishi K, Mano Y, et al. Predictors of extrahepatic recurrence after curative hepatectomy for hepatocellular carcinoma. Ann Surg Oncol. 2010 Oct;17(10):2740-6.

24 Takayama T, Makuuchi M, Hirohashi S, Sakamoto M, Yamamoto J, Shimada K, et al. Early hepatocellular carcinoma as an entity with a high rate of surgical cure. Hepatology. 1998 Nov;28(5):1241-6.

25 Kudo M. Targeted and immune therapies for hepatocellular carcinoma: predictions for 2019 and beyond. World J Gastroenterol. 2019 Feb;25(7):789-807.

26 Brown ZJ, Greten TF, Heinrich B. Adjuvant treatment of hepatocellular carcinoma: prospect of immunotherapy. Hepatology. 2019 Oct;70(4):1437-42.

27 Wang L, Wang W, Rong W, Li Z, Wu F, Liu $\mathrm{Y}$, et al. Postoperative adjuvant treatment strategy for hepatocellular carcinoma with microvascular invasion: a non-randomized interventional clinical study. BMC Cancer. 2020 Jul;20(1):614.

28 Tong H, Wei B, Chen S, Xie YM, Zhang MG, Zhang LH, et al. Adjuvant celecoxib and lanreotide following transarterial chemoembolisation for unresectable hepatocellular carcinoma: a randomized pilot study. Oncotarget. 2017 Jul;8(29):48303-12.

29 Sasaki K, Shindoh J, Margonis GA, Nishioka Y, Andreatos N, Sekine A, et al. Effect of background liver cirrhosis on outcomes of hepatectomy for hepatocellular carcinoma. JAMA Surg. 2017 Mar;152(3):e165059.

30 Takeishi K, Maeda T, Tsujita E, Yamashita Y, Harada N, Itoh S, et al. Predictors of intrahepatic multiple recurrences after curative hepatectomy for hepatocellular carcinoma. Anticancer Res. 2015;35(5):3061-6. 